\title{
The Weight Analysis of Northeast Passage Straits Index Based on AHP and Rough Set Theory
}

\author{
Zhe Wang ${ }^{1}$, Shanshan Ge ${ }^{1}$, Ren Zhang ${ }^{2}$, Mengqian Yang ${ }^{1}$, Deliang Jiang ${ }^{1}$ \\ ${ }^{1}$ Marine Meteorology Institute of PLA university of science and technology,Nanjing 211101 China \\ ${ }^{2}$ Meteorological disaster warning and forecast Assessment Collaborative Innovation Center \\ Nanjing University of information science and technology, Nanjing 210044 China
}

\section{基于层次分析法和粗粘集理论的东北航道海峡 指标权重分析}

王哲 ${ }^{1}$, 葛珊珊 ${ }^{1}$, 张韧 ${ }^{2}$, 杨孟倩 ${ }^{1}$, 姜德良 ${ }^{1}$

${ }^{1}$ 解放军理工大学 气象海洋学院, 江苏 南京 211101 , 中国

2 南京信息工程大学气象灾害预报预警与评估协同创新中心, 江苏 南京 210044 , 中国

\begin{abstract}
The index weight of the reasonable is the basis of objective and quantitative assessment of the risk. Taking a sample of the Northeast Passage Vilkitsky Strait, using the combination of subjectively and objectively, realize the complementary advantages of two ways, to establish the northeast passage natural environment risk index system, with the analytic hierarchy process to determine the subjective weight, rough set theory to determine the objective weight, organic integration of subjective and objective weights to get the combination weights, makes the northeast passage natural environment indicators of risk evaluation is more objective, so as to improve the accuracy of risk assessment. By calculating the combination weights, natural environment factor weight sorting get channel.
\end{abstract}

Keywords: AHP, set theory; the Northeast Passage; combining weights.

\begin{abstract}
摘要
指标权重的合理确定是风险的客观定量化 评估的基础。本文以东北航道维力基茨基海 峡等六个海峡为样本, 采用主观赋权与客观 赋权相结合的方式, 实现两种方式的优势互 补, 建立东北航道自然环境风险指标体系, 以层次分析法确定主观权重、粗糙集理论确 定客观权重, 将主、客观权重进行有机集成 得到组合权重, 使得东北航道自然环境风险 指标评价更具有客观性, 从而提高风险评估 的准确性。通过计算组合权重, 得到海峡自 然环境因素权重排序。
\end{abstract}

关键词: 层次分析法; 粗粘集理论; 东北航 道; 组合权重

1. 引言

全球变暖已是无可争议的事实, 变暖现象在 北极地区尤为明显, 其平均地面温度上升幅 度是全球温度上升幅度的两倍之多 [1]。北 极地区变暖会导致海冰迅速消融, 东北航线 的全线开通将成为现实。

风险研究从最开始的风险投资 [2]研 究到最后延伸到环境风险研究, 食品风险 
Risk Analysis and Crisis Response in Big Data Era (RAC-16)

研究 [3],目前国内学者围绕北极航道的战 略规划、自然环境变化、经济效益、政治环 境等方面开展了一系列研究: 李振福 [4]对 北极航线的中国战略进行了分析, 认为北极 海冰加速融化背景下, 中国应当采取 “争取” 战略，积极参与北极航线问题协调机制的制 定, 以获得相应的利益。李新情等 [5]分析 了东北航道关键海峡维利基茨基海峡及其 附近海域的通航性, 认为 8 月下旬到 10 月 中旬海峡适合通航, 为航行时间的选择提供 借鉴。王宇强 [6]等对东北航道的中-欧航线 经济性进行分析, 认为东北航道相比于传统 苏伊士运河航线缩短里程约 30\%, 具有巨大 经济前景。

东北航道的开通受到各个方面因素的 影响, 各领域学者都做了深入的研究, 但影 响东北航道开通与否的最关键因素是其自 然环境, 因此对其自然环境风险进行评估十 分必要。科学、准确的评估需要对指标因子 权重进行合理确定。本文将使用粗䊁集理论 与层次分析法相结合的方式，利用东北航道 六个关键海峡自然环境数据, 对评估对象的 自然环境因子指标权重进行确定。

\section{2. 数据和方法}

\section{1 研究区域介绍}

研究区域选取东北航道上六个关键海 峡, 分别是红军海峡、拉普捷夫海峡、维利 基茨基海峡、桑尼科夫海峡、咯拉海峡、德 朗海峡海峡, 六个海峡位置如图 1 所示。

\section{2 指标权重确定方法的选择}

权重确定方法有主观与客观两种, 其中 主观赋权法有特尔斐法 [7]、层次分析法 [8] 以及判断矩阵法 [9] 等; 客观赋权法主要有 粗粘集理论 [10]、神经网络法 [11] 等。

指标体系权重的科学、合理确定，对整 个系统的评估结果有直接影响。主观赋权法 往往对人的知识储备、科学素养、专业能力 等方面有很高的要求, 决策人员的主观意愿 往往会影响最终评价结果, 可能会导致与实 际情况有较大偏差; 客观赋权法虽然从数据 本身出发, 避免了人的影响因素, 但通常无 法考虑不同指标对评价目标的影响程度。本

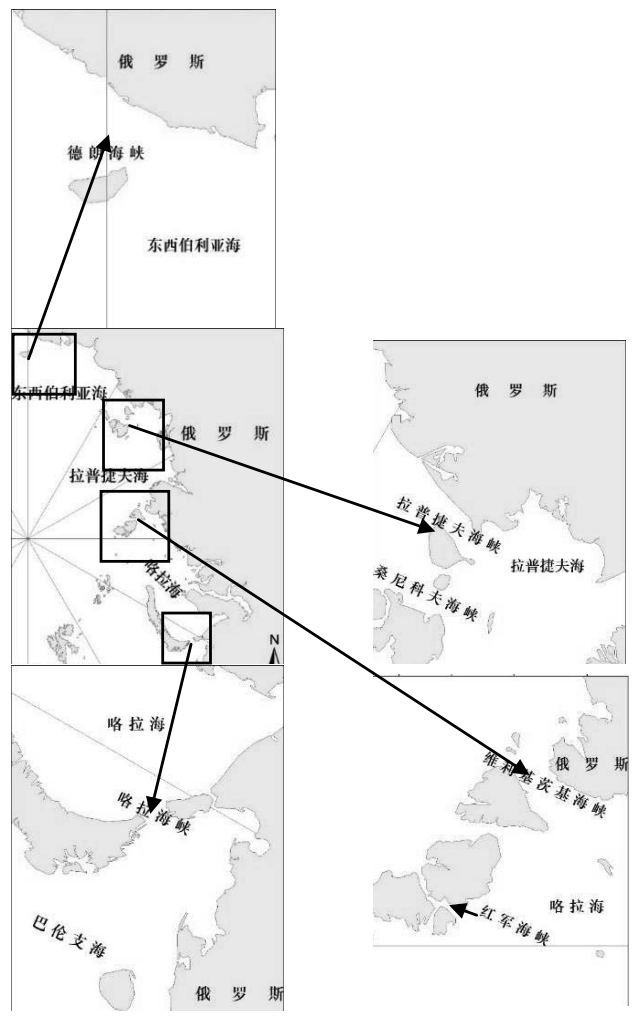

图 1 东北航道全景及各个海峡地理位置图

文充分考虑主观赋权与客观赋权两种方法 的优劣, 将主客观赋权方法相结合, 提出一 种主客观相结合的权重确定方法。

\section{3 层次分析法}

层次分析法是美国匹兹堡大学教授 T. L. Saaty 提出的一种方法, AHP 法的基本 思想: 将一个复杂无结构的问题分解为它的 各个组成部分; 将这些组成部分整理成为一 种递阶层次的顺序; 按照每个元素的相对重 要性赋予其表示主观判断的数量值; 然后综 合这些判断以决定到底哪个元素有着最大 的权重和如何影响问题的最终结果。利用层 次分析法进行决策的具体步骤为：1）建立递 阶层次结构模型；2）构造出各层次中的所有 判断矩阵；3）层次单排序及一致性检验；4) 层次总排序及一致性检验。 
Risk Analysis and Crisis Response in Big Data Era (RAC-16)

\section{4 粗楉集理论}

1982 年, 波兰数学家 Z. Pawlak 提出了 一种处理不完整和不精确知识的数据分析 理论, 该理论的核心概念是一个等价关系, 其所处理的属性值可为定量值, 也可以是定 性的。该理论称为粗糙集理论 (Rough set)。

定义 1: 称 $S=(U, A, V, f)$ 是一个信 息系统。U表示非空对象集, 即论域; $A$ 表示 属性的非空有限集, $\mathrm{A}=\mathrm{C} \cup \mathrm{D}, \mathrm{C} \cap \mathrm{D} \neq \varnothing$, $\mathrm{C}$ 称为条件属性集, $\mathrm{D}$ 称为决策属性集; $\mathrm{V}$ 是 属性值域; $\mathrm{f}$ 是 $\mathrm{U}$ 和 $\mathrm{A}$ 的关系集, 也称信息函 数集。若 $\mathrm{D}=\varnothing$, 则称信息系统 $\mathrm{S}$ 为数据表, 否则称为决策信息系统或简称决策表。

定义 2: 设 $\mathrm{R}$ 是 $\mathrm{U}$ 上的等价关系, 记为: $\operatorname{IND}(\mathrm{R})=\{(x, y) \in U \times U \mid \forall a \in A, f(x, a)$

$$
=f(y, a)\}
$$

$U / I N D(R)$ 称为 $U$ 的划分, 其中的任意元素称 为等价类。

定义 3：如果 $\operatorname{IND}(\mathrm{R})=\operatorname{IND}(\mathrm{R}-\{\mathrm{r}\})$ ， 称 $r$ 在 $R$ 中是可被约去的知识; 如果 $P=R-\{r\}$ 是独立的, 则 $P$ 是 $R$ 的一个知识约 简。所谓知识约简, 就是保持知识库分类能 力不变的条件下, 删除其中不相关或不重要 知识。

定义 4: 为了定义知识的近似程度, 引 用两个精确集概念-一下近似集和上近似 集:

$$
\begin{aligned}
& \bar{R}(\mathrm{X})=\{\mathrm{x} \in U \mid I N D(R) \subseteq \mathrm{X}\} \\
& \underline{R}(\mathrm{X})=\{\mathrm{x} \in U \mid I N D(R) \subseteq \mathrm{X}\}
\end{aligned}
$$

式中, $\bar{R}(\mathrm{X})$ 和 $R(\mathrm{X})$ 分别为 $\mathrm{X}$ 的 $\mathrm{R}$ 下近似集和 上近似集; 其中，下近似集又称为 $X$ 的 $R$ 正 域, 记为 $P O S_{R}(X)$ 。
策属性 $\mathrm{D}$ 的一个子集, 则决策表的近似度 $Y_{P}(Q)$ 可以表示为:

$$
\mathrm{V}_{\mathrm{P}}(\mathrm{Q})=\frac{\operatorname{POS}_{P}(Q)}{|U|}
$$

式中, $|\mathrm{U}|$ 和 $\left|\mathrm{POS}_{\mathrm{P}}(\mathrm{Q})\right|$ 分别表示各自的基, 即 所含元素的个数。

属性子集 $\mathrm{Ci} \subseteq \mathrm{C}$ 关于 $\mathrm{D}$ 的重要度 $\operatorname{Sig}_{\mathrm{D}}\left(C_{i}\right)$ 可以定义为:

$$
\operatorname{Sig}_{\mathrm{D}}\left(C_{i}\right)=\mathrm{Y}_{c}(D)-\mathrm{Y}_{C-C_{i}}(D)
$$

归一化处理得到海冰密度、温度、风速 和能见度以及无冰天数的权重为 0.2951 、 $0.2623 、 0.1311 、 0.1967 、 0.1148$ 。

\section{5 基于组合权重的评价公式确定}

$\omega_{\mathrm{oi}}$ 和 $\omega_{\mathrm{si}}$ 分别是属性 $X_{i}$ 主观权重和客观 权重, $\omega_{\mathrm{i}}$ 为两者的组合权重。其中 $\sum_{i=1}^{m} \omega_{\mathrm{oi}}=\sum_{i=1}^{m} \omega_{\mathrm{si}}=\sum_{i=1}^{m} \omega_{\mathrm{i}}=1,0 \leq$ $\omega_{\mathrm{oi}}, \omega_{\mathrm{si}}, \omega_{\mathrm{i}} \leq 1,(i=1,2, \ldots, m)$, 采用线形加 权组合法来确定评价指标的权重, 即:

$$
\omega_{i}=\mu * \omega_{o i}+(1-\mu) * \omega_{s i}
$$

对于线性加权组合法来说, 其关键是确 定主、客观权重的偏好系数 $\mu$ 。结合北极航 道气象资料贵乏这一现状, 本文中采用 0.618 标度法 [12], 认为主观赋权法较之于 客观赋权法稍微重要，取 $\mu=0.618$ 。

\section{3. 指标体系构建}

风险体系是由风险源危险性、承险体脆 弱性和防灾减灾能力综合作用而成, 北极地

\begin{tabular}{|c|c|c|c|}
\hline 目标层（A） & 准则层（B） & 一级指标层 (C) & 判别层 (D) \\
\hline $\begin{array}{l}\text { 东北航道自然 } \\
\text { 环境风险 }(A 1)\end{array}$ & 危险性（B1） & 海洋环境危险性 C1 & $\begin{array}{c}\text { 海冰密集度危险指数 D1 } \\
\text { 气温要素 D2 } \\
\text { 大风危险指数 D3 } \\
\text { 低能见度危险指数 D4 } \\
\text { 无冰以及清冰天数 D5 }\end{array}$ \\
\hline
\end{tabular}
区航行中, 海冰密度、风速、温度等要素对 航行安全性影响很大。构建东北航道自然风 险指标体系，如表 1 所示。

定义 5 : 令 $\mathrm{P}$ 和 $\mathrm{Q}$ 分别为条件属性 $\mathrm{C}$ 和决

表 1 东北航道自然环境风险指标体系 
Risk Analysis and Crisis Response in Big Data Era (RAC-16)

采用的海冰密集度、海平面风速等均采 用欧洲中期天气预报中心所发布的网格点 为 $0.125 \mathrm{o}$ 月平均资料。能见度数据来自美 国国家海洋和大气管理局网站, 水深数据采 用 ETOP01 高程数据。选取 2000 到 2014 年, 共 15 年的 $7 、 8 、 9 、 10$ 四个月份的月平均 气象数据, 作为指标权重确定的样本, 样本 数共 360 个。

\section{4. 指标权重确定}

\section{1 数据离散化处理和指标权重初步确定}

将各指标等级划分为“低”、“中”和“高”, 并对应的风险等级分别为 $1 、 2 、 3$ 。指标权 重的初步确定采用层次分析法, 邀请了 5 位 研究北极领域的相关专家学者打分并共同 商讨确定。各指标等级划分、评分规则和权 重见表 2。

表 2 评分规则表

\begin{tabular}{|c|c|c|c|c|}
\hline \multirow[t]{2}{*}{ 指标 } & \multicolumn{3}{|c|}{ 指标评分 } & \multirow[b]{2}{*}{ 权重 } \\
\hline & 高 & 中 & 低 & \\
\hline 海冰密度 & $>0.7$ & $0.7-0.4$ & $<0.4$ & 0.39 \\
\hline 风险等级 & 3 & 2 & 1 & \\
\hline 温度 ( $\left.{ }^{\circ} \mathrm{C}\right)$ & $<-5$ & $-5,-2$ & $>-2$ & 0.21 \\
\hline 风险等级 & 3 & 2 & 1 & \\
\hline 风速（m/s） & $>12.8$ & $12.8-7$ & $<7$ & 0.15 \\
\hline 风险等级 & 3 & 2 & 1 & \\
\hline 能见度 (m) & $<4000$ & $4000-6000$ & $>6000$ & 0.15 \\
\hline 风险等级 & 3 & 2 & 1 & \\
\hline 无冰天数 & $>0.8$ & $0.8-0.5$ & $<0.5$ & 0.1 \\
\hline
\end{tabular}

风险等级 3

\section{2 决策表构建与组合权重的确定}

以海峡自然风险指标为条件属性, 自然 风险综合等级为决策属性构建决策表, 其中 论域 $\mathrm{U}=\{1,2,3, \ldots \ldots, 360\}$, 条件属性集 $\mathrm{C}=\{\mathrm{C} 1, \mathrm{C} 2, \mathrm{C} 3, \mathrm{C} 4, \mathrm{C} 5\}$, 决策属性集 $\mathrm{D}=\{\mathrm{d}\}$ ， 其中: $\mathrm{C} 1$ 为海冰密度, $\mathrm{C} 2$ 为温度, $\mathrm{C} 3$ 为风

表 3 知识约简后的决策表

\begin{tabular}{lllllll}
\hline 序号 & 海冰密度 & 温度 & 风速 & 能见度 & 无冰天数 & 决策 \\
\hline 1 & 1 & 1 & 1 & 1 & 1 & 1 \\
2 & 1 & 1 & 1 & 1 & 2 & 1 \\
3 & 1 & 1 & 1 & 1 & 3 & 1 \\
4 & 1 & 1 & 1 & 1 & 1 & 3 \\
5 & 1 & 1 & 2 & 3 & 1 & 2 \\
6 & 1 & 2 & 1 & 1 & 1 & 1 \\
7 & 1 & 2 & 1 & 1 & 2 & 2 \\
8 & 1 & 2 & 1 & 1 & 3 & 2 \\
9 & 1 & 2 & 1 & 3 & 1 & 2 \\
10 & 1 & 1 & & & \\
\hline
\end{tabular}

对上面的数据论域分别按条件属性和 决策属性进行分类得:

条件属性分类:

$\mathrm{U} / \mathrm{IND}(\mathrm{C})$

$=\{\{1\},\{2\},\{3\},\{4\},\{5\},\{6\},\{7\},\{8\},\{9\}$,

$\{10\},\{11\},\{12\},\{13\},\{14\},\{15\},\{16\},\{17\}$,

$\{18\},\{19\},\{20\},\{21\},\{22\},\{23\},\{24\},\{25\}$,

$\{26\},\{27\},\{28\},\{29\},\{30\},\{31\},\{32\},\{33\}$,

$\{34\},\{35\},\{36\},\{37\}\}$
速, $\mathrm{C} 4$ 为能见度, $\mathrm{d}$ 为采用层次分析法初步 评价得到的自然风险综合等级。将数据进行 离散化处理和属性值的约简, 删除属性值相 同的样点后得到简化的决策表 (表 3), 由于 篇幅原因, 仅列出决策表 37 个样本的前 10 个。
决策属性分类：

$\mathrm{U} / \mathrm{IND}(\mathrm{D})=\{\{1,2,3,4,7,8,12\},\{6,9,1$ $0,11,13,14,15,16,17,18,19,20,21,22,23$ , 27, 29, 30\}, \{5, 24, 25, 26, 28, 31, 32, 33, 34

根据公式 (2)、(3)、(4) 可得各条件 属性集关于决策属性的近似精度:

$$
Y_{C}(D)=\frac{P O S_{C}(D)}{|U|}=1 、 Y_{C-\left\{C_{1}\right\}}(D)=
$$
, 35, 36, 37\} 
Risk Analysis and Crisis Response in Big Data Era (RAC-16)

$\frac{\operatorname{POS}_{C-C_{1}}(D)}{|U|}=\frac{19}{37} 、 Y_{C-\left\{C_{2}\right\}}(D)=\frac{\operatorname{POS}_{C-C_{2}}(D)}{|U|}=$

$\frac{21}{37}, \mathrm{Y}_{C-\left\{C_{3}\right\}}(D)=\frac{\operatorname{POS}_{C-C_{3}}(D)}{|U|}=\frac{29}{37} 、$

$Y_{C-\left\{C_{4}\right\}}(D)=\frac{P O S_{C-C_{4}}(D)}{|U|}=\frac{15}{37}, Y_{C-\left\{C_{5}\right\}}(D)=$

$\frac{\operatorname{POS}_{C-C_{5}}(D)}{|U|}=\frac{30}{37}$

根据公式 5 可得各条件属性关于决策属 性的重要度:

$$
\begin{gathered}
\sigma_{D}\left(C_{1}\right)=1-\gamma_{C-\left\{C_{1}\right\}}(D)=\frac{18}{37} 、 \\
\sigma_{D}\left(C_{2}\right)=1-\gamma_{C-\left\{C_{2}\right\}}(D)=\frac{16}{37} 、 \\
\sigma_{D}\left(C_{3}\right)=1-\gamma_{C-\left\{C_{3}\right\}}(D)=\frac{8}{37} 、 \\
\sigma_{D}\left(C_{4}\right)=1-\gamma_{C-\left\{C_{4}\right\}}(D)=\frac{12}{37} 、 \\
\sigma_{D}\left(C_{5}\right)=1-\gamma_{C-\left\{C_{5}\right\}}(D)=\frac{7}{37}
\end{gathered}
$$

归一化处理得到海冰密度、温度、风速 和能见度以及无冰天数的权重为 0.2951 、 $0.2623 、 0.1311 、 0.1967 、 0.1148$ 。将所得 的主客观权重代入式 (6) 可计算得到东北航 道自然环境风险因子的组合权重为分别为 海冰密度 0.35 , 温度 0.22 , 风速 0.14 , 能见 度 0.17 , 无冰天数 0.12 。

\section{5. 结论与讨论}

东北航道在北极海冰融化的大背景下, 开通前景日渐明朗, 可以预见在不久的将来 东北航道会迎来通航热潮, 东北航道相比于 传统航线, 有其独特的优势, 一方面避免了 海盗的侵扰，另一方面大大缩短了航程。东 北航道的通航环境研究可能会对国家东北 航道战略布局提供借鉴。本文基于大量气象、 地理等客观数据, 建立东北航道风险评估模 型, 采用主客观赋权法相结合的方式, 确定 风险指标权重, 得到如下两点重要结论:

（1）用层次分析法确定的主观权重, 粗鈢集方法确定的客观权重进行集成得到 组合权重, 既能克服层次分析法中由专家经 验造成的主观随意性, 又能弥补粗楉集理论 中无法体现各指标自身价值的重要性以及 各指标权重随样品的变化而变化的缺陷, 得
到更为合理、科学的权重结果, 为进一步进 行自然环境风险评估做铺垫。

（2）结果表明, 影响东北航道海峡自 然环境危险度的主要因素为海冰密度, 温度, 风速, 能见度, 无冰天数。一方面, 可以看出, 海冰密度是影响通航的最关键因素, 这与航 道自然环境实际情况基本符合, 并为科学合 理的确定海峡综合危险提供一定的依据。另 一方面, 通过分析不同因子权重排序, 可以 为实际航行决策提供辅助, 认清在东北航道 航行过程中的关键风险因子。因此, 将层次 分析法和粗䊁集理论确定的航道海峡自然 环境指标权重进行合理的组合将会集实际 结果、专家经验与航道实际为一体, 为科学 合理的海盗海峡自然环境风险定量评价奠 定基础。

目前为止, 国内学者对于北极航道通航 环境研究较少, 还需要投入更多的经历进行 进一步深入研究。对本文中的研究还存在一 些不足: 一方面, 评估过程中应当选取更加 合理的自然风险评估指标, 既要考虑指标对 评估结果的影响程度, 也要考虑指标数据的 可获取性, 提高模型的科学性和有效性; 另 一方面, 东北航道各个海峡地理环境等条件 各不相同, 建立模型时, 应当结合不同海峡 实际, 选取合适的指标, 提高模型的有效性。

北极海冰的融化以及东北航道的开通, 必将对现有的国际贸易体系和政治环境产 生深刻的影响, 不同学科背景的专家学者应 当充分发挥各自学科优势, 对北极展开全方 位、多角度的研究, 未雨绸缪, 为将来中国 能够在北极事务处理中占得先机, 取得应有 的利益。

\section{参考文献}

[1] Screen J A, Simmonds I. The central role of diminishing sea ice in recent Arctic temperature amplification. Nature, 2010, 464(7293):1334-7.

[2] Yunfei Li, Zongfang Zhou Research on Model for Evaluating Risks of Venture Capital Projects. Journal of Risk Analysis and Crisis Response, Vol. 1, No. 2 (November 2011), 142-148. 
[3] Jingyuan Yin, Haiyan Gao, Wei Pei. A Study on Food Safety Risk Analysis under the Condition of Traditional Agriculture. Journal of Risk Analysis and Crisis Response, Vol. 1, No. 2 (November 2011), 118-125.

[4] 李振福. 北极航线的中国战略分析. 中 国软科学, 2009(1):1-7.

[5] 李新情, 慈天宇, 罗斯瀚, 等. 北极东北 航道维利基茨基海峡海冰时空变化及 适航性分. 极地研究, 2015(3):282-288.

[6] 王宇强, 寿建敏. 航经“东北航道”的中 一欧航线设计及经济性分析. 航海技 术, 2013(2):21-24.

[7] Zhang B, Zhang Y, Chen D, White R E, Li Y. A quantitative evaluation system of soil productivity for intensive agriculture in China. Geoderma, 2004, 123(3): 319-331.

[8] Ying X, Zeng G, Chen G, Tang L, Wang K, Huang D. Combining AHP with GIS in synthetic evaluation of eco -environment quality - A case study of Hunan Province, China. Ecological Modelling, 2007, 209(2): 97-109.

[9] 张吴, 陶然,李志勇,杜华. 判断矩阵法在 网页恶意脚本检测中的应用. 兵工学报, 2008, 29(4):469-473.

[10] Pawlak Z . Rough sets. International Journal of Computer Information Science, 1982, 11(5):342-356.

[11] 刘东海,梁国庆,周卫,王秀斌,夏文建.基 于神经网络的土壤肥力综合评价. 中国 土壤与肥料,2011,(5):12-19.

[12] 程爱宝, 古德生, 刘洪强. 基于 AHP 与粗 糙集理论的采空区稳定性影响因素权 重分析. 中国安全生产科学技术, 2011, 07(9):50-55. 